\title{
The Role of Tirzepatide, Dual GIP and GLP-1 Receptor Agonist, in the Management of Type 2 Diabetes: The SURPASS Clinical Trials
}

\author{
Thinzar Min (D) - Stephen C. Bain
}

Received: October 27, 2020 / Accepted: November 28, 2020

(C) The Author(s) 2020

\begin{abstract}
Glucagon-like peptide 1 (GLP-1) based therapy is an established treatment option for the management of type 2 diabetes mellitus (T2DM) and is recommended early in the treatment algorithm owing to glycaemic efficacy, weight reduction and favourable cardiovascular outcomes. Glucose-dependent insulinotropic polypeptide (GIP), on the other hand, was thought to have no potential as a glucose-lowering therapy because of observations showing no insulinotropic effect from supraphysiological infusion in people with T2DM. However, emerging evidence has illustrated that co-infusion of GLP-1 and GIP has a synergetic effect, resulting in significantly increased insulin response and glucagonostatic response, compared with separate administration of each hormone. These observations have
\end{abstract}

T. Min $(\varangle) \cdot$ S. C. Bain

Diabetes Research Group, Swansea University

Medical School, Swansea SA2 8PP, UK

e-mail: thinzar.min@swansea.ac.uk

T. Min

Department of Diabetes and Endocrinology, Neath Port Talbot Hospital, Swansea Bay University Health Board, Swansea SA12 7BX, UK

S. C. Bain

Department of Diabetes and Endocrinology, Singleton Hospital, Swansea Bay University Health Board, Swansea SA2 8QA, UK led to the development of a dual GIP/GLP-1 receptor agonist, known as a 'twincretin'. Tirzepatide is a novel dual GIP/GLP-1 receptor agonist formulated as a synthetic peptide containing 39 amino acids, based on the native GIP sequence. Pre-clinical trials and phase 1 and 2 clinical trials indicate that tirzepatide has potent glucose lowering and weight loss with adverse effects comparable to those of established GLP-1 receptor agonists. The long-term efficacy, safety and cardiovascular outcomes of tirzepatide will be investigated in the SURPASS phase 3 clinical trial programme. In this paper, we will review the pre-clinical and phase 1 and 2 trials for tirzepatide in the management of T2DM and give an overview of the SURPASS clinical trials.

Keywords: Glucagon-like peptide 1; Glucosedependent insulinotropic polypeptide; Incretin hormone; Type 2 diabetes; Tirzepatide 


\section{Key Summary Points}

Tirzepatide is a dual GIP/GLP-1 receptor agonist (RA) formulated as a synthetic linear peptide containing 39 amino acids, based on the native GIP sequence.

Tirzepatide is administered subcutaneously once weekly because of its half-life of 5 days.

In the phase 1 and phase 2 trials tirzepatide demonstrated dose-dependent reduction in HbA1c (up to 2.4\%) and body weight (up to $11.3 \mathrm{~kg}$ ) in patients with T2DM.

The clinical efficacy of tirzepatide is superior to the GLP-1 RA dulaglutide.

Most common adverse effects are related to the gastrointestinal tract and comparable to the GLP-1 RA dulaglutide.

There are nine ongoing SURPASS clinical trials investigating the efficacy and safety of tirzepatide in individuals with T2DM on various glucose-lowering therapies.

The SURPASS-CVOT is a large phase 3 , randomised, double-blind, cardiovascular outcomes trial for tirzepatide assessing both non-inferiority and superiority of tirzepatide against dulaglutide $(1.5 \mathrm{mg}$ weekly).

\section{DIGITAL FEATURES}

This article is published with digital features to facilitate understanding of the article. You can access the digital features on the article's associated Figshare page. To view digital features for this article go to https://doi.org/10.6084/m9. figshare.13293128.

\section{INTRODUCTION}

Type 2 diabetes mellitus (T2DM) is a chronic progressive disease associated with microvascular and macrovascular complications and increased cardiovascular mortality. The pathogenesis of T2DM is a complex process and yet to be fully understood. A combination of insulin resistance (in the early phase of the disease process) and relative insulin deficiency results in hyperglycaemia. Chronic hyperglycaemia leads to glucose toxicity, which in turn causes pancreatic beta cell dysfunction and exacerbates insulin deficiency in the late phase. The ultimate result of this is a viscous cycle of hyperglycaemia leading to a worsening metabolic state. One of the main modifiable risk factors for diabetes is obesity. The parallel rise in the prevalence of obesity and T2DM (known as 'diabesity') is a global health challenge. Obesity is strongly associated with insulin resistance, one of the key features in pathogenesis of T2DM and one of the barriers for achieving good glycaemic control. Management of T2DM, therefore, includes dietary intervention and lifestyle modification to promote weight loss along with pharmacotherapy to combat hyperglycaemia and optimise metabolic parameters such as blood pressure and lipids, and metabolic surgery in some cases.

The ideal glucose-lowering medication should be efficacious, not associated with weight gain (but promoting weight loss), have low risk of hypoglycaemia and proven cardiovascular benefits. The last 15 years or so have seen new classes of diabetes medications available for management of T2DM: glucagon-like peptide 1 (GLP-1) receptor agonists (exenatide being the first, approved by the US Food and Drug Administration [FDA] in 2005), dipeptidyl peptidase 4 (DPP4) inhibitors (sitagliptin, first DPP4i, approved in 2006) and sodium-glucose co-transporter 2 (SGLT2) inhibitors (canagliflozin [USA] and dapagliflozin [EU] in 2012 and 2013 respectively). These newer glucose-lowering drugs are effective (HbA1c reduction $1.5-1.8 \%$ with once weekly semaglutide $1 \mathrm{mg}$ ) and promote weight loss (4.5-6.5 kg loss with once weekly semaglutide $1 \mathrm{mg}$ ) [1]. Despite 
these advances in therapy, there are a significant proportion of people with T2DM who do not achieve optimal glycaemic control. As per the National Diabetes Audit data, only $65.9 \%$ of people with T2DM in England and Wales achieved the recommended HbA1c $<58 \mathrm{mmol} / \mathrm{mol}(7.5 \%)$ [2]. The NDA data also showed that $85 \%$ of people with T2DM were overweight or obese. Therefore, there is a drive for the development of glucoselowering medications which are not only effective in reduction of HbA1c but also in promoting substantial weight loss.

This article is based on previously conducted studies and does not contain any new studies with human participants or animals performed by any of the authors.

\section{PHYSIOLOGY OF INCRETIN HORMONES}

The term 'incretin', first described in 1932, refers to hormones released from the gut that regulate the insulin response to a meal [3]. The seminal paper by Elrick et al., published in 1964, demonstrated a significant and sustained rise in plasma insulin response following an oral glucose load, compared to a parental glucose load [4]. This phenomenon was later known as 'the incretin effect' and this accounts for up to $65 \%$ of postprandial insulin secretion $[5,6]$. There are two principal incretin hormones responsible for the incretin effect: glucagon-like peptide 1 (GLP-1) and glucose-dependent insulinotropic polypeptide (GIP, formerly known as gastric inhibitory polypeptide) (Table 1) [7].

GLP-1 is a 30 amino acid peptide, synthesised in the L cells of the intestine. Once released into the circulation, GLP-1 binds to a specific GLP-1 receptor, which is expressed in the pancreas, gastrointestinal tract, kidney, heart and brain [7]. Endogenous GLP-1 has a short half-life of $1-2$ min and is metabolised by the enzyme DPP4. In additional to the wellknown insulinotropic property in response to nutrients in the gut, GLP-1 has a glucagonostatic effect, inhibiting glucagon secretion in the hyperglycaemic and normoglycaemic state but not during hypoglycaemia. Extrapancreatic properties of GLP-1 include slowing of gastric emptying, promoting satiety and reducing food intake [8].

Table 1 Functions of GLP-1 and GIP in glucose and energy metabolism

\begin{tabular}{|c|c|c|c|}
\hline & & GLP-1 & GIP \\
\hline \multirow[t]{5}{*}{ Pancreas } & Beta cells & $\uparrow$ Insulin synthesis & $\uparrow$ Insulin synthesis \\
\hline & & $\uparrow$ Insulin secretion & $\uparrow$ Insulin secretion \\
\hline & & $\uparrow$ Cell proliferation & $\uparrow$ Cell proliferation \\
\hline & & $\uparrow$ Glucose sensing & $\uparrow$ Glucose sensing \\
\hline & Alpha cells & $\downarrow$ Glucagon secretion & $\uparrow$ Glucagon secretion \\
\hline \multirow[t]{2}{*}{ Brain } & & $\uparrow$ Satiety & \\
\hline & & $\downarrow$ Appetite & \\
\hline \multirow[t]{2}{*}{ Gastrointestinal } & & $\downarrow$ GI motility & \\
\hline & & $\downarrow$ Gastric emptying & \\
\hline \multirow[t]{3}{*}{ Adipose tissues } & & & $\uparrow$ Lipolysis \\
\hline & & & $\uparrow$ Fatty acid synthesis \\
\hline & & & ?Anti-lipogenic effect \\
\hline
\end{tabular}


GIP is a 4 amino acid peptide, produced by the $\mathrm{K}$ cells of the intestine, mainly located in the duodenum and proximal jejunum. GIP is released in response to oral nutrients, especially carbohydrates and lipids. GIP, like GLP-1, has a short half-life (4-7 min) and is inactivated by DPP4, shortly after release. GIP receptors are present in various tissues such as the pancreas, adipose tissue, gastric mucosa, heart, adrenal cortex, bone and brain [7]. Endogenous GIP also stimulates glucose-dependent insulin secretion and is responsible for a greater proportion of the incretin effect than GLP-1 $[9,10]$. One difference between GIP and GLP-1 is the effect on glucagon secretion. Unlike GLP-1, GIP has dual functions: a glucagonotropic property in the normoglycaemic and hypoglycaemic state, and glucagonostatic in the hyperglycaemic state [11]. The effect of GIP on adipose tissue and body weight regulation has yet to be established. Some studies reported that GIP receptor knockout mice, unlike control mice, were resistant to diet-induced obesity [12], suggesting the adipogenic effect, whereas other studies demonstrated that chronic elevation of GIP concentrations in a transgenic mouse reduced diet-induced obesity and promoted insulin sensitivity, glucose tolerance and beta cell function [13]. Since GIP is found to have glucagonotropic properties, the other hypothetical mechanism for GIP-induced weight loss is through the anorexic and anti-lipogenic effect of glucagon [11].

\section{INCRETIN HORMONES IN THE PATHOPHYSIOLOGY OF T2DM}

The incretin effect is significantly reduced in patients with T2DM, compared to people without diabetes [7]. The proposed mechanisms behind the loss of incretin effect include (1) a reduction in incretin hormone response to nutrients (hyposecretion) and (2) a reduction in insulinotropic effect on pancreatic beta cells. Early studies showed that there was hyposecretion of GLP-1 in people with T2DM $[14,15]$, but emerging evidence does not support this observation [16]. In fact, reduction in insulinotropic effect of GLP-1 was observed in patients with T2DM [17] and supraphysiological doses of GLP-1 receptor agonists were found to partly restore the incretin effect. This has led to the development of widely available GLP-1based therapy for management of T2DM.

With respect to GIP secretion, there are consistent reports demonstrating no reduction in GIP secretion in people with T2DM [18-20]. However, GIP resistance, a complete loss of insulinotropic effect of GIP at physiological concentrations as well as supraphysiological concentrations was observed in people with T2DM $[17,21]$. In addition, some studies found that the glucagonotropic effect of GIP persists even in the hyperglycaemic state [22]. These observations in the past have made GIP unattractive as a therapeutic agent for T2DM. However, emerging evidence suggests that GIP resistance can be modified and restored by improving glycaemic control. GIP receptors on pancreatic beta cells are downregulated owing to chronic hyperglycaemia, resulting in subsequent loss of insulinotropic effect. In a study by Hojberg et al., GIP administration increased insulin response, partly restoring insulinotropic properties in eight people with T2DM whose glycaemic control was optimised by using 4 -week insulin therapy [23]. A potential additional benefit of GIP is safeguarding against hypoglycaemia. GIP infusion was associated with lower glucose infusion requirement to maintain a desired level of blood glucose during an insulin-induced hypoglycaemic clamp [24]. The evidence mentioned above has paved the way to development of GIP receptor agonistbased therapy for T2DM.

\section{DUAL GIP AND GLP-1 AGONISM}

Co-administration of GIP and a GLP-1 receptor agonist (RA) in healthy individuals has a additive effect, generating a significantly increased insulin response compared with separate administration of each hormone $[9,25]$. Furthermore, the combined infusion produced a significant glucagonostatic effect while separate administration of GIP or GLP-1 did not suppress glucagon secretion more than glucose alone [25]. However, 
in people with T2DM, co-administration of GIP and GLP-1 over a short period of time (4-6 h) did not generate an insulin response greater than that of GLP-1 administration alone [26-28].

The long-term effect of GIP and GLP-1 receptor agonism was first described by Finan et al. who developed a unimolecular dual agonist of GIP and GLP-1 receptors, referred to as a 'twincretin' [29]. The twincretin was shown to have high affinity to GLP-1 and GIP receptors with negligible glucagon receptor activity. In animal studies, GIP and GLP-1 receptor co-agonist therapy at 1-3 weeks produced a dose-dependent reduction in blood glucose, body weight, food intake and fat mass compared to placebo, equimolar dose of exendin-4 or liraglutide. The co-agonist can be modified by attaching a polyethylene glycol (PEG) or a 16-carbon acyl chain to extend half-life (allowing weekly dosing). The PEGylated co-agonist was investigated in 44 people with T2DM. After 6 weeks, a dose-dependent decrease in HbA1c compared to placebo was observed. The co-agonist was well tolerated with mild to moderate GI side effects and no hypoglycaemic events [29].

\section{TIRZEPATIDE}

Tirzepatide (LY3298176) developed by Eli Lilly is a dual GIP/GLP-1 receptor agonist formulated as a synthetic linear peptide containing 39 amino acids, based on the native GIP sequence. It is attached to a 20-carbon fatty diacid moiety, which binds to albumin, prolonging its half-life to 5 days and thus enabling once weekly dosing. Tirzepatide has a comparable GIP receptor binding affinity to native GIP and five times lower GLP1 receptor affinity than that of native GLP-1 [30]. Similar to the GLP-1 RA tirzepatide is administered subcutaneously. The clinical efficacy, safety and tolerability of tirzepatide has been reported in phase 1 and phase 2 clinical trials (Table 2).

\section{EFFICACY OF TIRZEPATIDE IN PHASE 1 CLINICAL TRIALS}

A phase 1 proof-of-concept clinical trial was conducted in 53 people with T2DM, comprising five groups: two fixed dose groups (tirzepatide $0.5 \mathrm{mg}$ and $5.0 \mathrm{mg}$ ) and two dose-titration groups (tirzepatide $0.5 / 5 / 10 / 10 \mathrm{mg}$ and $0.5 / 5 /$ $10 / 15 \mathrm{mg}$ ) and a placebo group. The study had 4 weeks treatment followed by 4 weeks safety follow-up. Baseline characteristics of study participants were mean age $56.8 \pm 6.9$ years, $53 \%$ male, $77 \%$ White, 13\% Asian, 8\% Black, BMI $31.2 \pm 4.0 \mathrm{~kg} / \mathrm{m}^{2}$ and HbA1c $8.4 \pm 0.8 \%$ (all values in mean $\pm \mathrm{SD}$ ). After 4 weeks treatment, a statistically significant dose-dependent reduction in HbA1c was observed in both titration groups compared to placebo. Fasting glucose and fasting serum insulin were significantly decreased in both titration groups compared to placebo. All tirzepatide treatment doses except $0.5 \mathrm{mg}$ reduced the glucose responses (glucose area under the curve [AUC]) during the oral glucose tolerance test (OGTT) compared to placebo. Similarly, 7-point selfmonitoring of blood glucose demonstrated reduction in postprandial glucose levels in a dose-dependent manner. Similar to the HbA1c reduction, a dose-dependent reduction in body weight in tirzepatide groups compared to the placebo group was observed. The 0.5/5/10/ $10 \mathrm{mg}$ and $0.5 / 5 / 10 / 15 \mathrm{mg}$ groups achieved $2.39 \mathrm{~kg}$ and $2.95 \mathrm{~kg}$ weight loss respectively [30].

A similar phase 1 placebo-controlled, randomised study of tirzepatide was conducted in 48 Japanese people with T2DM. The treatment arm of the Japanese study consisted of one fixed (5 mg) dose group and two dose-titration groups (2.5 mg for 2 weeks, $5 \mathrm{mg}$ for 2 weeks and $10 \mathrm{mg}$ for 4 weeks; and $5 \mathrm{mg}$ for 2 weeks, $10 \mathrm{mg}$ for 4 weeks and $15 \mathrm{mg}$ for 2 weeks). The study had a treatment exposure of 8 weeks with a 4-weeks safety follow-up. Baseline characteristics were mean age $57.4 \pm 8.8$ years, $47 \%$ male, BMI $25.4 \pm 3.2 \mathrm{~kg} / \mathrm{m}^{2}$ and HbA1c $8.0 \pm 0.8 \%$. In line with the proof-of-concept study, compared to placebo there was a significant reduction in HbA1c from baseline in all tirzepatide groups ($1.62 \%,-1.78 \%,-2.05 \%$ in $5 \mathrm{mg}, 10 \mathrm{mg}$, $15 \mathrm{mg}$ tirzepatide respectively). Significant reduction in body weight was also noted in all three tirzepatide groups (weight loss of $1.9 \mathrm{~kg}$, $3.6 \mathrm{~kg}, 5.1 \mathrm{~kg}$ in $5 \mathrm{mg}, 10 \mathrm{mg}, 15 \mathrm{mg}$ tirzepatide respectively) [31]. 


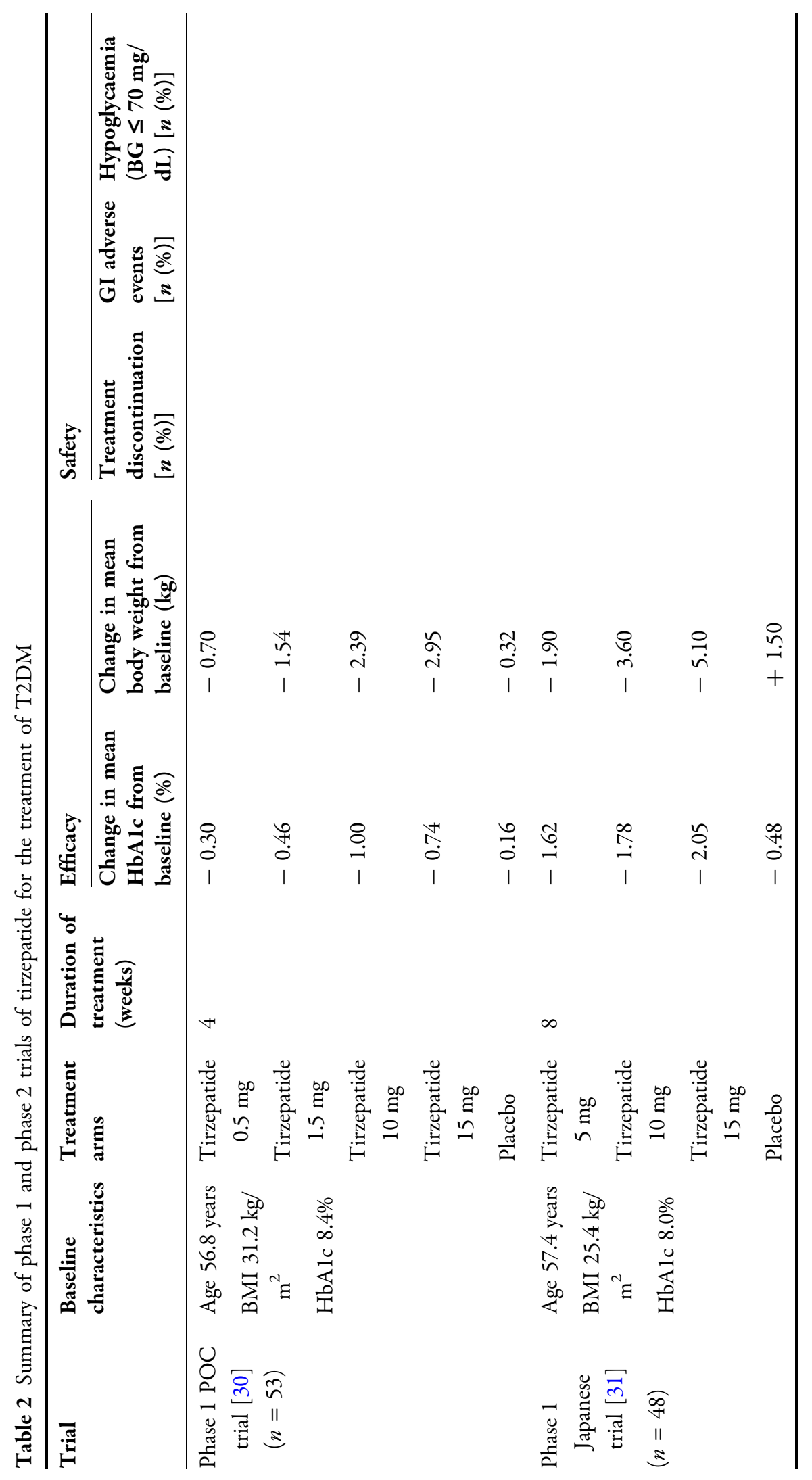




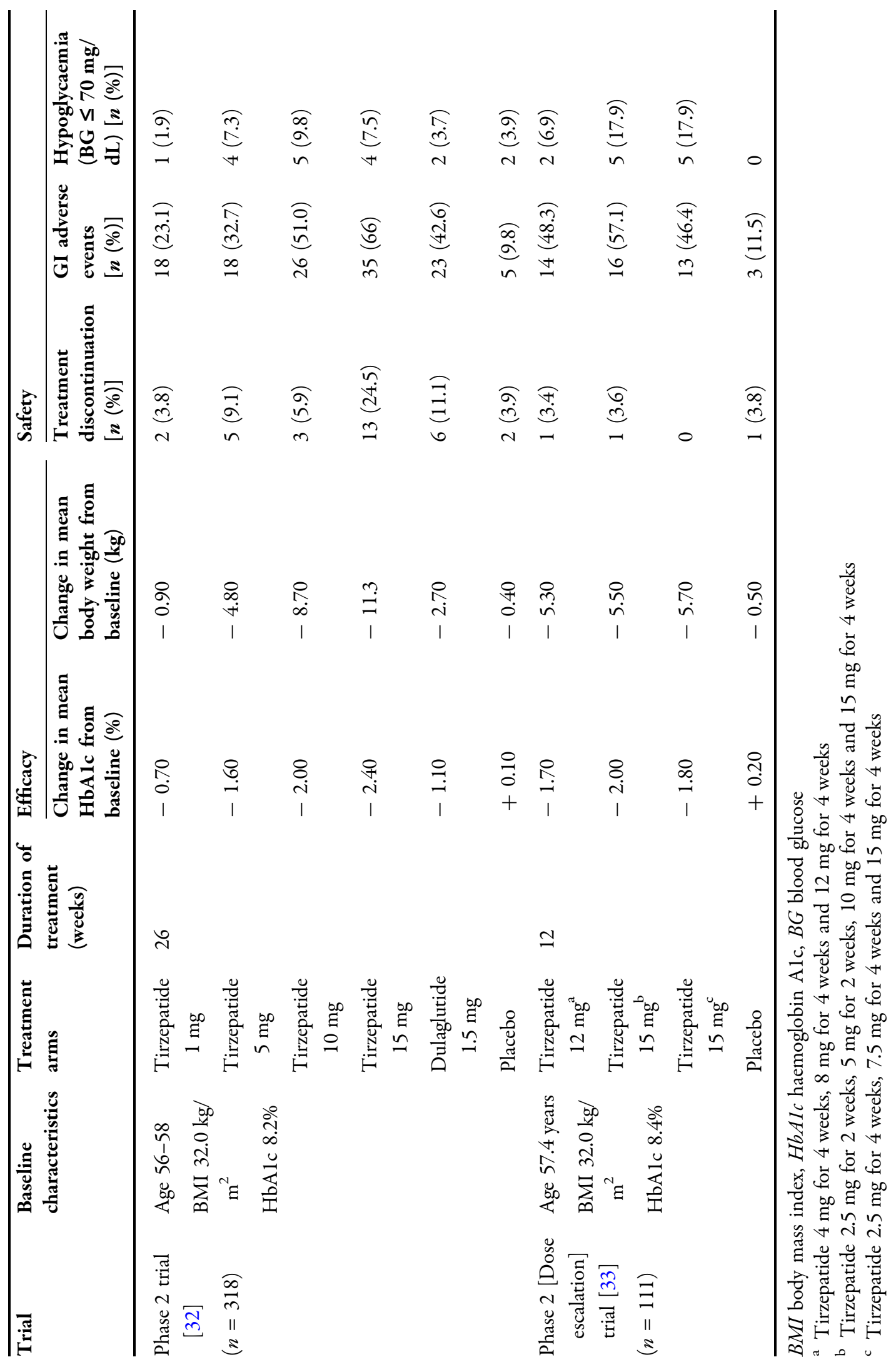




\section{EFFICACY OF TIRZEPATIDE IN PHASE 2 CLINICAL TRIALS}

The phase 2 trial of tirzepatide was a 26-week, placebo-controlled, double-blind, randomised trial in 318 people with T2DM treated with lifestyle intervention alone with or without metformin monotherapy. The trial consisted of two fixed dose ( $1 \mathrm{mg}$ and $5 \mathrm{mg}$ ) tirzepatide groups and two dose-titration groups $(5 \mathrm{mg}$ for 2 weeks and $10 \mathrm{mg}$ for the remaining trial period; and $5 \mathrm{mg}$ for 2 weeks, $10 \mathrm{mg}$ for 4 weeks and $15 \mathrm{mg}$ for the remaining trial period). There was also a dulaglutide $1.5 \mathrm{mg}$ group and a placebo group. Baseline characteristics were mean age $57 \pm 9$ years, $53 \%$ male, BMI $32.6 \pm 5.9 \mathrm{~kg} /$ $\mathrm{m}^{2}$, duration of diabetes $9 \pm 6$ years and HbA1c $8.1 \% \pm 1 \%$. Approximately $90 \%$ of subjects received metformin at baseline and this continued throughout the trial period. In line with the phase 1 clinical trials, the reduction in HbA1c and fasting plasma glucose with tirzepatide was dose dependent (HbA1c reduction from baseline of $8 \%$ by $0.7 \%, 1.6 \%, 2.0 \%$ and $2.4 \%$ with $1 \mathrm{mg}, 5 \mathrm{mg}, 10 \mathrm{mg}$ and $15 \mathrm{mg}$ tirzepatide doses, respectively). The $5 \mathrm{mg}, 10 \mathrm{mg}$ and $15 \mathrm{mg}$ tirzepatide doses demonstrated a greater reduction in HbA1c at 26 weeks, compared with dulaglutide $1.5 \mathrm{mg}$. The proportion of patients who achieved HbA1c targets of $<7.0 \%$ and $<6.5 \%$ were higher in all tirzepatide groups except $1 \mathrm{mg}$ dose $(90 \%$ and $82 \%$ achieved HbA1c of $<7 \%$ and $<6.5 \%$, respectively, in the tirzepatide $10 \mathrm{mg}$ group while $52 \%$ and $39 \%$ achieved HabA1c of $<7 \%$ and $<6.5 \%$, respectively, in the dulaglutide group. Tirzepatide also resulted in significant weight loss during the trial period. Mean weight change from baseline was $-0.9 \mathrm{~kg},-4.8 \mathrm{~kg},-8.7 \mathrm{~kg}$ and $-11.3 \mathrm{~kg}$ in $1 \mathrm{mg}, 5 \mathrm{mg}, 10 \mathrm{mg}$ and $15 \mathrm{mg}$ tirzepatide groups compared to $-0.4 \mathrm{~kg}$ for placebo and $-2.7 \mathrm{~kg}$ for dulaglutide. The proportion of patients who achieved $>5 \%,>10 \%$ and $>15 \%$ weight loss was $71 \%, 39 \%$ and $25 \%$, respectively, in the $10 \mathrm{mg}$ tirzepatide group; $62 \%, 38 \%$ and $22 \%$, respectively, in the $15 \mathrm{mg}$ tirzepatide group; $22 \%$ and $9 \%$ of the dulaglutide group achieved weight reduction of $>5 \%$ and $>10 \%$ respectively [32].
Another phase 2 clinical trial with similar objectives was designed to assess three dose-titration regimens of tirzepatide in people with T2DM who received lifestyle intervention with or without metformin therapy. The three dose regimens were (1) the $12 \mathrm{mg}$ group, $(n=29)$ [ $4 \mathrm{mg}$ for 4 weeks, $8 \mathrm{mg}$ for 4 weeks and $12 \mathrm{mg}$ for 4 weeks]; (2) the $15 \mathrm{mg}-1$ group, $(n=28)$ [2.5 mg for 2 weeks, $5 \mathrm{mg}$ for 2 weeks, $10 \mathrm{mg}$ for 4 weeks and $15 \mathrm{mg}$ for 4 weeks]; and (3) the $15 \mathrm{mg}$-2 group, $(n=28)$ [2.5 mg for 4 weeks, $7.5 \mathrm{mg}$ for 4 weeks and $15 \mathrm{mg}$ for 4 weeks]. After 12 weeks of treatment, significant reductions in $\mathrm{HbA1c}$, fasting plasma glucose and body weight were observed in all three treatment regimens, compared to placebo. Mean HbA1c changes from baseline ranged from $-1.7 \%$ to $-2.0 \%$ and mean body weight changes ranged from $5.3 \mathrm{~kg}$ to $-5.7 \mathrm{~kg}$ in the tirzepatide groups [33].

\section{SAFETY AND TOLERABILITY OF TIRZEPATIDE}

Since tirzepatide is a dual GIP/GLP-1 agonist, the side effect profile is comparable to that of a GLP-1 receptor agonist. The most frequently observed side effects were related to the gastrointestinal (GI) system and nausea, diarrhoea and vomiting were the most common adverse events. The incidence of GI adverse events was $23.1 \%$ for $1 \mathrm{mg}, 32.7 \%$ for $5 \mathrm{mg}, 51.0 \%$ for $10 \mathrm{mg}$ and $66.0 \%$ for $15 \mathrm{mg}$, demonstrating a dose-dependent behaviour (versus $42.6 \%$ for dulaglutide and $9.8 \%$ for placebo). The second most common adverse effect was reduced appetite, the incidence ranging from $3.8 \%$ to $18.9 \%$ in tirzepatide-treated groups. Study drug discontinuation due to adverse events was greater with the higher dose of tirzepatide $(25 \%$ with the $15 \mathrm{mg}$ group, $9.1 \%$ with the $10 \mathrm{mg}$ group and $5.1 \%$ with the $5 \mathrm{mg}$ group); the discontinuation rate for dulaglutide was $11.1 \%$ [32].

The titration-regimen trial also demonstrated the dose-dependent incidence of GI adverse events: $48.3 \%, 57.1 \%$ and $46.4 \%$ in the tirzepatide $12 \mathrm{mg}, 15 \mathrm{mg}-1$ and $15 \mathrm{mg}-2$ groups, respectively, and $11.5 \%$ with placebo. Prevalence of study drug discontinuation due to 
adverse events was 3.4\% (1 patient), 3.6\% (1 patient), no patient and $3.8 \%$ (1 patient) in the tirzepatide $12 \mathrm{mg}, 15 \mathrm{mg}-1,15 \mathrm{mg}-2$ and placebo groups, respectively. These findings contrasted with those of the 26-week phase 2 clinical trial where a greater prevalence of study drug discontinuation occurred in the higher dose group. The study authors suggested that a lower starting dose and small subsequent dose increments were associated with lower incidence of GI adverse events [33]. The severity of GI adverse events in both trials was mild to moderate.

With regard to hypoglycaemia, the incidence of hypoglycaemia was low in both phase 2 trials and comparable across the treatment groups. In the 26-week trial, the prevalence of hypoglycaemia was $9.8 \%$ and $7.5 \%$ in the $10 \mathrm{mg}$ and $15 \mathrm{mg}$ groups, respectively [32]. In the 12-week trial, $6.9 \%$ in the $12 \mathrm{mg}$ group, $17.9 \%$ in the $15 \mathrm{mg}-1$ group and $17.9 \%$ in the $15 \mathrm{mg}-2$ groups reported hypoglycaemia [33]. There were no reports of severe hypoglycaemia in either study.

Other adverse events included pancreatitis (two patients in the tirzepatide $5 \mathrm{mg}$ group), cholecystitis (one patient in the tirzepatide $10 \mathrm{mg}$ group and one in the dulaglutide $1.5 \mathrm{mg}$ group), injection site reactions (2-8\% in tirzepatide groups, $11.1 \%$ in the dulaglutide group and $3.9 \%$ in the placebo group), hypersensitivity and antibody formation (3\% in tirzepatide groups, $9.8 \%$ in the placebo group and none in the dulaglutide group) [32].

\section{OVERVIEW OF THE SURPASS CLINICAL TRIALS}

The SURPASS clinical trial programme aimed to assess the efficacy and safety of tirzepatide as a treatment to improve glycaemic control in people with T2DM (Table 3) [34-42]. The SURPASS phase 3 clinical trials include six global, two Japanese and one Asia-Pacific studies. These trials include anti-hyperglycaemic therapy-naïve patients (patients treated with diet and lifestyle alone) as well as patients on various oral anti-hyperglycaemic agents (metformin, sulfonylurea, pioglitazone, SGLT2 inhibitor and/or insulin). Some studies are placebo- controlled, and others have active comparators, such as GLP-1 RAs (dulaglutide and semaglutide), long-acting insulin analogues (glargine and degludec) or short-acting insulin analogue (lispro). The SURPASS trials are designed to assess once weekly tirzepatide doses of $5 \mathrm{mg}$, $10 \mathrm{mg}$ and $15 \mathrm{mg}$. Based on the results of the phase 2 trials, a dose-escalation algorithm is used in the phase 3 trials as follows: starting dose at $2.5 \mathrm{mg}$ weekly for initial 4 weeks, then the dose increment of $2.5 \mathrm{mg}$ every 4 weeks until the maintenance dose of $5 \mathrm{mg}, 10 \mathrm{mg}$ or $15 \mathrm{mg}$ is reached. Therefore, it takes 4 weeks to reach the $5 \mathrm{mg}$ dose, 12 weeks for the $10 \mathrm{mg}$ dose and 20 weeks for the $15 \mathrm{mg}$ dose. The primary endpoint for each of the studies (except SURPASS J-combo) is change in HbA1c from baseline. The primary endpoint of the SURPASS $\mathrm{J}$ combo is the number of participants with more than one severe adverse event.

SURPASS-CVOT is a large phase 3, randomised, double-blind, cardiovascular outcomes trial for tirzepatide assessing both noninferiority and superiority of tirzepatide against dulaglutide (1.5 mg weekly). The SURPASSCVOT will include 12,500 participants with T2DM (aged 40 years, HbA1c between $7.5 \%$ and $10.5 \%, \quad B M I \geq 25 \mathrm{~kg} / \mathrm{m}^{2}$ ) and established atherosclerotic cardiovascular disease from 30 countries. In addition to study drug, participants should continue to receive standard medical care for diabetes which include concomitant oral glucose-lowering therapy and/ or various insulin regimens. Participants who have treatment with any GLP-1 RA or pramlintide in 3 months prior to screening visit and who discontinue a GLP-1 RA or pramlintide because of intolerability are excluded from the study. Participants with sever heart failure (NYHA class 4) and those with end-stage renal disease or on dialysis are excluded from the study. The use of SGLT2 inhibitors at baseline is one of the stratification factors for randomisation. Tirzepatide is started at $2.5 \mathrm{mg}$ weekly and to be escalated to $15 \mathrm{mg}$ weekly or to the highest maintenance dose tolerated by the study participant. The dose of dulaglutide is initiated and maintained at $1.5 \mathrm{mg}$ weekly. A sham dose escalation of dulaglutide will be employed to maintain the double-blind study design. The 


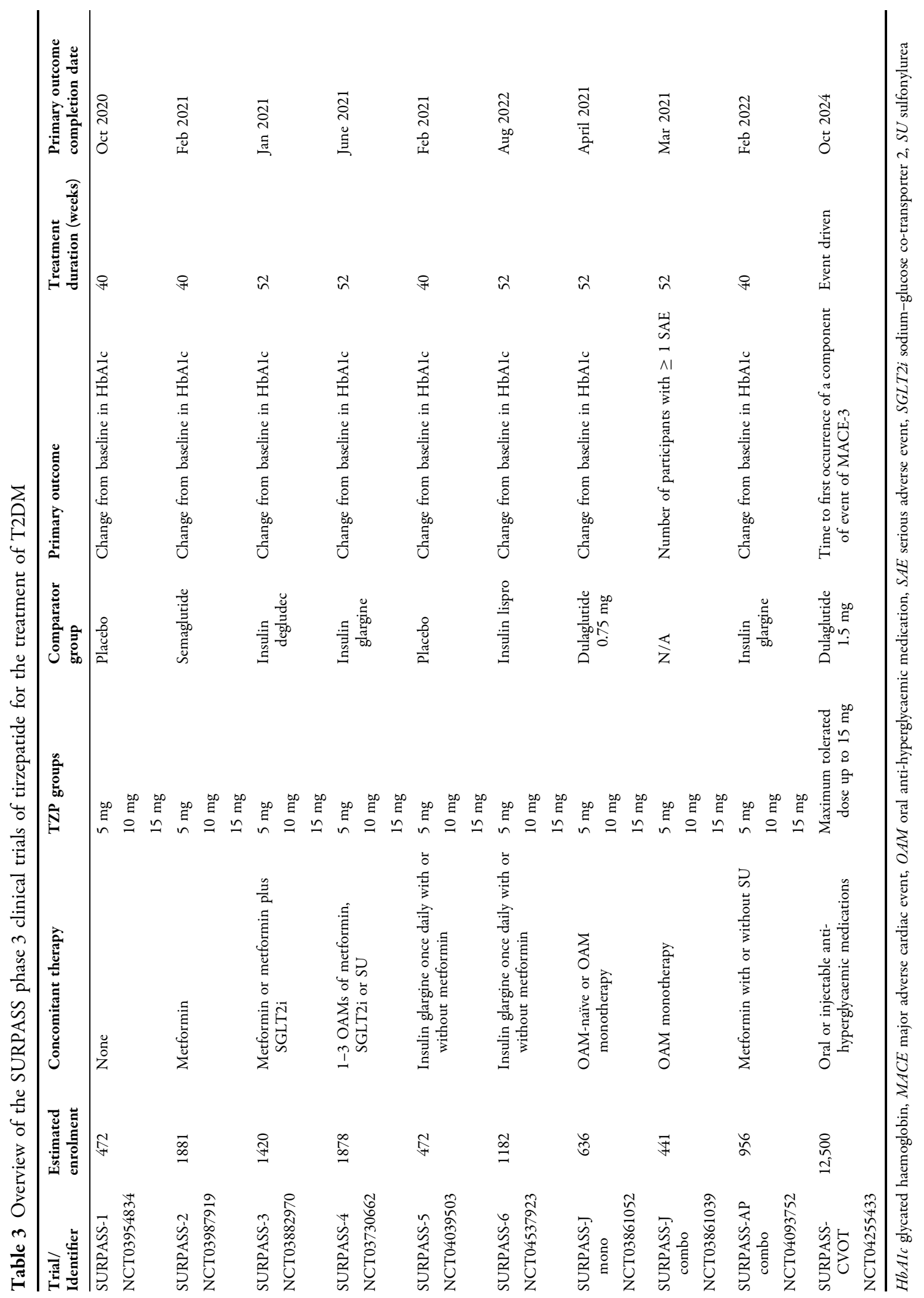


primary outcome measure is time to first occurrence of death from cardiovascular (CV) causes, non-fatal myocardial infarction (MI) or non-fatal stroke (MACE-3). Secondary outcome measures include time to all-cause mortality, time to occurrence of each component of the primary endpoint, time to first occurrence of new or worsening nephropathy and change in body weight, HbA1c, urine albumin to creatinine ratio and lipid profile from baseline. The study is event-driven and is expected to run for approximately 54 months (randomisation up to study completion).

\section{CLINICAL SIGNIFICANCE OF THE SURPASS CLINICAL TRIALS}

Diabetes care has evolved over the last two decades, shifting from a glucocentric approach to multifactorial intervention with the aim of minimising CV morbidity and mortality. International guidelines recommend the choice of anti-hyperglycaemic therapy based not only on the degree of hyperglycaemia but also on the presence or absence of CV disease, renal disease and obesity. GLP-1 RAs and SGLT2 inhibitors have gained popularity due to their clinical efficacy and favourable CV outcomes. Despite these developments, there are still unmet needs for people living with diabetes, especially those with obesity. Tirzepatide has demonstrated greater $\mathrm{HbA} 1 \mathrm{c}$ reduction ( $-2.4 \%$ with $15 \mathrm{mg}$ tirzepatide) and superior weight loss $(-11.3 \mathrm{~kg}$ with tirzepatide $15 \mathrm{mg}$ ) with comparable adverse effects versus the GLP-1 RA dulaglutide in phase 2 trials. Of note, semaglutide, currently regarded as the most potent GLP-1 RA, demonstrated $1.5-1.8 \% \mathrm{HbA1c}$ reduction and $4.5-6.5 \mathrm{~kg}$ weight reduction [1]. It is not possible to compare the efficacy of tirzepatide and semaglutide with available data from phase 2 trials; however, the SURPASS- 2 trial will directly compare the efficacy and safety of tirzepatide versus semaglutide with the potential for superiority of tirzepatide, particularly with respect to weight reduction. Since the prevalence of diabesity is increasing, diabetes medications with potent weight loss will be increasingly attractive in modern diabetes care. Amongst available
GLP-1 RAs, liraglutide $3.0 \mathrm{mg}$ daily subcutaneous injection (Saxenda ${ }^{\circledR}$ ) has approval for chronic weight management in patients with obesity or with a weight-related comorbidity and a BMI $\geq 27 \mathrm{~kg} / \mathrm{m}^{2}$; however, for the management of T2DM, the approval licence remains at the once daily dose of $1.8 \mathrm{mg}$ [43]. In the SCALE trial which compared liraglutide $3 \mathrm{mg}$ versus $1.8 \mathrm{mg}$ versus placebo, there was a small but significant reduction in weight and HbA1c in the liraglutide $3 \mathrm{mg}$ group compared to liraglutide $1.8 \mathrm{mg}$ group [44]. However, the SCALE study was not designed to compare the efficacy of liraglutide $3 \mathrm{mg}$ versus $1.8 \mathrm{mg}$. In the SCALE insulin trial, liraglutide $3 \mathrm{mg}$ was associated with a HbA1c reduction of $1.1 \%$ compared to placebo (0.6\%) [45]. Of interest, a higher dose of dulaglutide for glucose lowering was recently approved by the FDA [46] following the AWARD-11 trial which demonstrated HbA1c reduction of $1.9 \%$ and $1.7 \%$ and weight reduction of $5 \mathrm{~kg}$ and $4.3 \mathrm{~kg}$ with dulaglutide $4.5 \mathrm{mg}$ and $3 \mathrm{mg}$ QW respectively [47].

Some SURPASS clinical trials include SGLT2 inhibitors as concomitant therapy (e.g. SURPASS-3 and -4). SGLT2 inhibitors have a favourable effect on weight and CVD outcomes. It would be interesting to see the combined effect of tirzepatide and SGLT2 inhibitors on efficacy and CVD outcomes. Of interest, the combined use of once weekly exenatide and dapagliflozin resulted in superior $\mathrm{HbA1c}$ reduction and greater weight loss compared to the exenatide group and the dapagliflozin group [48]. Therefore, one can argue that tirzepatide might not be solely responsible for favourable results. None of the SURPASS trials are designed to compare tirzepatide against SGLT2 inhibitors. Further study comparing tirzepatide against SGLT2 inhibitors would be of use in clinical decision-making. Since the 2008 publication of FDA guidance on anti-hyperglycaemic therapy, medications for diabetes should not only be efficacious in reduction in HbA1c but should also have favourable CV outcomes (at least no negative CV impact). The SURPASSCVOT trial will provide long-term data on the $\mathrm{CV}$ safety as well as efficacy of tirzepatide. 


\section{OTHER CLINICAL TRIALS OF TIRZEPATIDE}

Tirzepatide has also been investigated for the management of obesity in individuals without T2DM. SURMOUNT-1, a phase 3, randomised, double-blind, placebo-controlled trial, is ongoing and plans to recruit 2400 participants with obesity and comorbidity excluding T2DM (those with BMI $\geq 30 \mathrm{~kg} / \mathrm{m}^{2}$, or $\geq 27 \mathrm{~kg} / \mathrm{m}^{2}$ and previous diagnosis with at least one of the following comorbidities: hypertension, dyslipidaemia, obstructive sleep apnoea, cardiovascular disease). The primary endpoints are change in body weight from baseline and percentage of participants achieving at least 5\% weight loss after 72 weeks [49]. SYNERGY-NASH is a randomized, double-blind, placebo-controlled phase 2 study comparing the efficacy and safety of tirzepatide in patients with nonalcoholic steatohepatitis (NASH) [50].

\section{CONCLUSION}

Tirzepatide, a dual GIP/GLP-1 receptor agonist, is a new incretin-based therapy for type 2 diabetes. The degree of $\mathrm{HbA1c}$ reduction and weight reduction observed in pre-clinical, phase 1 and 2 clinical trials has not previously been observed in diabetes clinical trials. The comprehensive phase 3 SURPASS clinical trial programme will confirm comparable efficacy, safety and cardiovascular outcomes of tirzepatide in the management of T2DM diabetes. The SURPASS trials will also provide insight into understanding of incretin hormones, particularly the role of GIP in energy metabolism. The SURPASS-1 trial is due to be completed in October 2020 and results are eagerly awaited.

\section{ACKNOWLEDGEMENTS}

Funding. No funding or sponsorship was received for publication of this article.
Authorship. All named authors meet the International Committee of Medical Journal Editors (ICMJE) criteria for authorship for this manuscript, take responsibility for the integrity of the work as a whole, and have given final approval for the version to be published.

Disclosures. Dr Thinzar Min reports personal fees and travel grants from AstraZeneca, Boehringer Ingelheim, and Napp. Professor Stephen C Bain reports grants and personal fees from AstraZeneca, Novo Nordisk, and SanofiAventis; personal fees from Boehringer Ingelheim, Eli Lilly, and Merck Sharp \& Dohme; grants from Medscape; expert advice provided to All-Wales Medicines Strategy Group and National Institute for Health and Care Excellence UK; and partnership in Glycosmedia.

Compliance with Ethics Guidelines. This article is based on previously conducted studies and does not contain any new studies with human participants or animals performed by any of the authors.

Data Availability. Data sharing is not applicable to this article as no datasets were generated or analyzed during the current study.

Open Access. This article is licensed under a Creative Commons Attribution-NonCommercial 4.0 International License, which permits any non-commercial use, sharing, adaptation, distribution and reproduction in any medium or format, as long as you give appropriate credit to the original author(s) and the source, provide a link to the Creative Commons licence, and indicate if changes were made. The images or other third party material in this article are included in the article's Creative Commons licence, unless indicated otherwise in a credit line to the material. If material is not included in the article's Creative Commons licence and your intended use is not permitted by statutory regulation or exceeds the permitted use, you will need to obtain permission directly from the copyright holder. To view a copy of this licence, visit http://creativecommons.org/licenses/by$\mathrm{nc} / 4.0 /$. 


\section{REFERENCES}

1. Aroda VR, Ahmann A, Cariou B, et al. Comparative efficacy, safety, and cardiovascular outcomes with once-weekly subcutaneous semaglutide in the treatment of type 2 diabetes: insights from the SUSTAIN 1-7 trials. Diabetes Metab. 2019;45(5): 409-18.

2. NDA. National Diabetes Audit, 2017-18. Report 1: care processes and treatment targets. England and Wales. https://files.digital.nhs.uk/88/F1E544/ National\%20Diabetes\%20Audit\%202017-18\% 20Full\%20Report $\% 201 \% 2$ C $\% 20$ Care $\%$ 20Processes\%20and\%20Treatment\%20Targets.pdf. 2019. Accessed 18 Sept 2020.

3. Kim W, Egan JM. The role of incretins in glucose homeostasis and diabetes treatment. Pharmacol Rev. 2008;60(4):470-512.

4. Elrick H, Stimmler L, Hlad CJ Jr, Arai Y. Plasma insulin response to oral and intravenous glucose administration. J Clin Endocrinol Metab. 1964;24: 1076-82.

5. Creutzfeldt W. The incretin concept today. Diabetologia. 1979;16(2):75-85.

6. Nauck MA, Homberger E, Siegel EG, et al. Incretin effects of increasing glucose loads in man calculated from venous insulin and C-peptide responses. J Clin Endocrinol Metab. 1986;63(2):492-8.

7. Nauck MA, Meier JJ. The incretin effect in healthy individuals and those with type 2 diabetes: physiology, pathophysiology, and response to therapeutic interventions. Lancet Diabetes Endocrinol. 2016;4(6):525-36.

8. Drucker DJ, Nauck MA. The incretin system: glucagon-like peptide-1 receptor agonists and dipeptidyl peptidase- 4 inhibitors in type 2 diabetes. Lancet. 2006;368(9548):1696-705.

9. Nauck MA, Bartels E, Orskov C, Ebert R, Creutzfeldt W. Additive insulinotropic effects of exogenous synthetic human gastric inhibitory polypeptide and glucagon-like peptide-1-(7-36) amide infused at near-physiological insulinotropic hormone and glucose concentrations. J Clin Endocrinol Metab. 1993;76(4):912-7.

10. Gasbjerg LS, Helsted MM, Hartmann B, et al. Separate and combined glucometabolic effects of endogenous glucose-dependent insulinotropic polypeptide and glucagon-like peptide 1 in healthy individuals. Diabetes. 2019;68(5):906-17.

11. Christensen M, Vedtofte L, Holst JJ, Vilsboll T, Knop FK. Glucose-dependent insulinotropic polypeptide: a bifunctional glucose-dependent regulator of glucagon and insulin secretion in humans. Diabetes. 2011;60(12):3103-9.

12. Miyawaki K, Yamada $\mathrm{Y}$, Ban N, et al. Inhibition of gastric inhibitory polypeptide signaling prevents obesity. Nat Med. 2002;8(7):738-42.

13. Kim SJ, Nian C, Karunakaran S, Clee SM, Isales CM, McIntosh CH. GIP-overexpressing mice demonstrate reduced diet-induced obesity and steatosis, and improved glucose homeostasis. PLoS One. 2012;7(7):e40156.

14. Vilsboll T, Krarup T, Deacon CF, Madsbad S, Holst JJ. Reduced postprandial concentrations of intact biologically active glucagon-like peptide 1 in type 2 diabetic patients. Diabetes. 2001;50(3):609-13.

15. Toft-Nielsen MB, Damholt MB, Madsbad S, et al. Determinants of the impaired secretion of glucagon-like peptide- 1 in type 2 diabetic patients. J Clin Endocrinol Metab. 2001;86(8):3717-23.

16. Calanna S, Christensen M, Holst JJ, et al. Secretion of glucagon-like peptide- 1 in patients with type 2 diabetes mellitus: systematic review and metaanalyses of clinical studies. Diabetologia. 2013;56(5):965-72.

17. Nauck MA, Heimesaat MM, Orskov C, Holst JJ, Ebert R, Creutzfeldt W. Preserved incretin activity of glucagon-like peptide 1 [7-36 amide] but not of synthetic human gastric inhibitory polypeptide in patients with type- 2 diabetes mellitus. J Clin Invest. 1993;91(1):301-7.

18. Vilsboll T, Krarup T, Sonne J, et al. Incretin secretion in relation to meal size and body weight in healthy subjects and people with type 1 and type 2 diabetes mellitus. J Clin Endocrinol Metab. 2003;88(6):2706-13.

19. Calanna S, Christensen M, Holst JJ, et al. Secretion of glucose-dependent insulinotropic polypeptide in patients with type 2 diabetes: systematic review and meta-analysis of clinical studies. Diabetes Care. 2013;36(10):3346-52.

20. Holst JJ, Windelov JA, Boer GA, et al. Searching for the physiological role of glucose-dependent insulinotropic polypeptide. J Diabetes Investig. 2016;7(Suppl 1):8-12.

21. Vilsboll T, Krarup T, Madsbad S, Holst JJ. Defective amplification of the late phase insulin response to glucose by GIP in obese type II diabetic patients. Diabetologia. 2002;45(8):1111-9.

22. Chia CW, Carlson OD, Kim W, et al. Exogenous glucose-dependent insulinotropic polypeptide 
worsens post prandial hyperglycemia in type 2 diabetes. Diabetes. 2009;58(6):1342-9.

23. Hojberg PV, Vilsboll T, Rabol R, et al. Four weeks of near-normalisation of blood glucose improves the insulin response to glucagon-like peptide-1 and glucose-dependent insulinotropic polypeptide in patients with type 2 diabetes. Diabetologia. 2009;52(2):199-207.

24. Christensen MB, Calanna S, Holst JJ, Vilsboll T, Knop FK. Glucose-dependent insulinotropic polypeptide: blood glucose stabilizing effects in patients with type 2 diabetes. J Clin Endocrinol Metab. 2014;99(3):E418-26.

25. Elahi D, McAloon-Dyke M, Fukagawa NK, et al. The insulinotropic actions of glucose-dependent insulinotropic polypeptide (GIP) and glucagon-like peptide-1 (7-37) in normal and diabetic subjects. Regul Pept. 1994;51(1):63-74.

26. Lund A, Vilsboll T, Bagger JI, Holst JJ, Knop FK. The separate and combined impact of the intestinal hormones, GIP, GLP-1, and GLP-2, on glucagon secretion in type 2 diabetes. Am J Physiol Endocrinol Metab. 2011;300(6):E1038-46.

27. Daousi C, Wilding JP, Aditya S, et al. Effects of peripheral administration of synthetic human glucose-dependent insulinotropic peptide (GIP) on energy expenditure and subjective appetite sensations in healthy normal weight subjects and obese patients with type 2 diabetes. Clin Endocrinol (Oxf). 2009;71(2):195-201.

28. Mentis N, Vardarli I, Kothe LD, et al. GIP does not potentiate the antidiabetic effects of GLP-1 in hyperglycemic patients with type 2 diabetes. Diabetes. 2011;60(4):1270-6.

29. Finan B, Ma T, Ottaway N, et al. Unimolecular dual incretins maximize metabolic benefits in rodents, monkeys, and humans. Sci Transl Med. 2013;5(209):209ra151.

30. Coskun T, Sloop KW, Loghin C, et al. LY3298176, a novel dual GIP and GLP-1 receptor agonist for the treatment of type 2 diabetes mellitus: from discovery to clinical proof of concept. Mol Metab. 2018;18:3-14.

31. Ohwaki K, Furihata K, Mimura M, Oura T, Imaoka T. 1024-P: effect of tirzepatide, a dual GIP and GLP1 receptor agonist, on glycemic control and body weight in Japanese patients with T2DM. Diabetes. 2019;68(Supplement 1).

32. Frias JP, Nauck MA, Van J, et al. Efficacy and safety of LY3298176, a novel dual GIP and GLP-1 receptor agonist, in patients with type 2 diabetes: a randomised, placebo-controlled and active comparator-controlled phase 2 trial. Lancet. 2018;392(10160):2180-93.

33. Frias JP, Nauck MA, Van J, et al. Efficacy and tolerability of tirzepatide, a dual glucose-dependent insulinotropic peptide and glucagon-like peptide- 1 receptor agonist in patients with type 2 diabetes: a 12-week, randomized, double-blind, placebo-controlled study to evaluate different dose-escalation regimens. Diabetes Obes Metab. 2020;22(6):938-46.

34. ClinicalTrial.gov. A study of tirzepatide (LY3298176) compared to dulaglutide in participants with type 2 diabetes (SURPASS J-mono). https://clinicaltrials.gov/ct2/show/NCT03861052. 2019. Accessed 18 Sept 2020.

35. ClinicalTrial.gov. A study of tirzepatide (LY3298176) compared with dulaglutide on major cardiovascular events in participants with type 2 diabetes (SURPASS-CVOT). https://clinicaltrials. gov/ct2/show/NCT04255433. 2019. Accessed 18 Sept 2020.

36. ClinicalTrial.gov. A study of tirzepatide (LY3298176) in participants with type 2 diabetes not controlled with diet and exercise alone (SURPASS-1). https://clinicaltrials.gov/ct2/show/record/ NCT03954834. 2019. Accessed 18 Sept 2020.

37. ClinicalTrial.gov. A study of tirzepatide (LY3298176) in participants with type 2 diabetes on metformin with or without sulfonylurea (SURPASSAP-combo). https://clinicaltrials.gov/ct2/show/ NCT04093752. 2019. Accessed 18 Sept 2020.

38. ClinicalTrial.gov. A study of tirzepatide (LY3298176) once a week versus insulin glargine once a day in participants with type 2 diabetes and increased cardiovascular risk (SURPASS-4). https:// clinicaltrials.gov/ct2/show/NCT03730662. 2019. Accessed 18 Sept 2020.

39. ClinicalTrial.gov. A study of tirzepatide (LY3298176) versus insulin degludec in participants with type 2 diabetes (SURPASS-3). https:// clinicaltrials.gov/ct2/show/NCT03882970. 2019. Accessed 18 Sept 2020.

40. ClinicalTrial.gov. A study of tirzepatide (LY3298176) versus placebo in participants with type 2 diabetes inadequately controlled on insulin glargine with or without metformin (SURPASS-5). https://clinicaltrials.gov/ct2/show/NCT04039503. 2019. Accessed 18 Sept 2020.

41. ClinicalTrial.gov. A study of tirzepatide (LY3298176) versus semaglutide once weekly as add-on therapy to metformin in participants with type 2 diabetes (SURPASS-2). https://clinicaltrials. gov/ct2/show/NCT03987919. 2019. Accessed 18 Sept 2020. 
42. ClinicalTrial.gov. A study of tirzepatide (LY3298176) versus insulin lispro (U100) in participants with type 2 diabetes inadequately controlled on insulin glargine (U100) with or without metformin (SURPASS-6). https://clinicaltrials.gov/ct2/ show/NCT04537923?term=SURPASS\&cond= Diabetes+Mellitus\%2C+Type+2\&draw=2\&rank=3. 2019. Accessed 18 Sept 2020.

43. BNF. Liraglutide. https://bnf.nice.org.uk/drug/ liraglutide.html. 2020. Accessed 9 Oct 2020.

44. Davies MJ, Bergenstal R, Bode B, et al. Efficacy of liraglutide for weight loss among patients with type 2 diabetes: the SCALE diabetes randomized clinical trial. JAMA. 2015;314(7):687-99.

45. Garvey WT, Birkenfeld AL, Dicker D, et al. Efficacy and safety of liraglutide $3.0 \mathrm{mg}$ in individuals with overweight or obesity and type 2 diabetes treated with basal insulin: the SCALE insulin randomized controlled trial. Diabetes Care. 2020;43(5):1085-93.

46. https://investor.lilly.com/news-releases/newsrelease-details/fda-approves-additional-dosestrulicityr-dulaglutide-treatment. 2020. Accessed 9 Oct 2020.
47. Frias JP, Bonora E, Nevarez Ruiz LA, et al. 357-OR: efficacy and safety of dulaglutide $3 \mathrm{mg}$ and $4.5 \mathrm{mg}$ vs. dulaglutide $1.5 \mathrm{mg}$ : 52-week results from AWARD-11. Diabetes. 2020;69. https://doi.org/10. 2337/db20-357-OR.

48. Frias JP, Guja C, Hardy E, et al. Exenatide once weekly plus dapagliflozin once daily versus exenatide or dapagliflozin alone in patients with type 2 diabetes inadequately controlled with metformin monotherapy (DURATION-8): a 28 week, multicentre, double-blind, phase 3 , randomised controlled trial. Lancet Diabetes Endocrinol. 2016;4(12):1004-16.

49. ClinicalTrial.gov. A study of tirzepatide (LY3298176) in participants with obesity or overweight (SURMOUNT-1). https://clinicaltrials.gov/ ct2/show/NCT04184622. 2019. Accessed 20 Nov 20 .

50. ClincalTrial.gov. A study of tirzepatide (LY3298176) in participants with nonalcoholic steatohepatitis (NASH) (SYNERGY-NASH). https://clinicaltrials. gov/ct2/show/NCT04166773. 2019. Accessed 20 Nov 20. 